\title{
Métodos de evaluación de desnutrición intrahospitalaria en niños
}

\section{Screening tools in hospitalized children}

\author{
Rigoberto García-Rivera, ${ }^{1}$ Ericka Montijo-Barrios, ${ }^{2}$ Roberto Cervantes-Bustamante, ${ }^{3}$ Flora Zárate-Mondragón, ${ }^{1}$ \\ José Francisco Cadena-León, ${ }^{1}$ Erick Manuel Toro-Monjaraz, ${ }^{1}$ Monserrat Cázares-Méndez, ${ }^{1}$ Martha López- \\ Ugalde, ${ }^{1}$ Jaime Alfonso Ramírez-Mayans, ${ }^{4}$ Mariana Roldán-Montijo ${ }^{5}$
}

\begin{abstract}
Resumen
OBJETIVO: Describir las distintas herramientas de cribado para el diagnóstico de desnutrición intrahospitalaria y comparar sus diferencias.

MATERIAL Y MÉTODO: Estudio comparativo y retrospectivo de búsqueda bibliográfica en las bases de datos Scientific Electronic Library Online (SciELO), Red Informática de Medicina Avanzada (RIMA), Plataforma HIGHWIRE, Literatura Latinoamericana y del Caribe en Ciencias de la Salud (LILACS) y Medline. Las palabras clave de búsqueda fueron: herramientas de cribado nutricional, desnutrición intrahospitalaria y diagnóstico de malnutrición intrahospitalaria.

RESULTADOS: Se encontraron seis herramientas de cribado pero ninguna estandarizada para la edad que valoran, miden diferentes parámetros y evalúan de distintas formas el riesgo de desnutrición intrahospitalaria. Ninguna puede considerarse patrón de referencia ni tampoco son comparables entre sí. La sensibilidad y especificidad que publica cada una de las herramientas no sustentan que alguna pruebe mejor o peor pronóstico en niños hospitalizados.

CONCLUSIONES: Si bien todas las herramientas estudiadas pretenden orientar la conducta médica para lograr intervenciones dietéticas oportunas, todas son subjetivas y sumamente específicas para el lugar en el que se aplican. Desde luego que se requiere la estandarización que atienda las necesidades propias del lugar en donde van a aplicarse. El Instituto Nacional de Pediatría requiere una herramienta específica para las condiciones en que se labora en este hospital; por tanto, y con base en la variabilidad de las herramientas comparadas, se propone diseñar una herramienta de fácil aplicación que permita evaluar oportuna y rápidamente el riesgo nutricional de nuestros pacientes. PALABRAS CLAVE: Malnutrición; niños hospitalizados.
\end{abstract}

\section{Abstract}

OBJECTIVE: To describe and compare the screening tools for hospitalized children.

MATERIAL AND METHOD: Bibliographic search was performed in Scientific Electronic Library Online (SciELO), Red Informática de Medicina Avanzada (RIMA), Plataforma HIGHWIRE, Literatura Latinoamericana y del Caribe en Ciencias de la Salud (LILACS), U.S. National Library of Medicine (Medline). The key words were nutritional screening, hospitalized malnutrition and diagnosis in hospitalized malnutrition. We reviewed all the literature and present this article.

RESULTS: The most popular stools used in children are Nutritional Risk Score (NRS) Tool, Simple Pediatric Nutritional Risk Score (SPNRS), Subjective Global Nutritional Assessmet for Children (SGNA), Screening Tool for Assessment of Malnutrition in Paediatrics (STAMP), Screening Tool for Risk On Nutritional status and Growth (STRONGkids), and Paediatric Yorkhill Malnutrition Score (PYMS). The stools were not standardized in age, have different scores and each one evaluates different things, that's why we do not have a gold standard and it is not possible to compare them. The sensibility and specificity of each stool have do not demonstrated acutely malnourished.

CONCLUSIONS: All the stools revised pretend to determine risk of malnutrition in hospitalized children to do opportune interventions, but almost all the stools are subjective and they are so specific for the place they applied it. The tools may be more standardize and must be adapted to each hospital. We suggest to do a tool easy to be applied and responding to the hospital and specific population.

KEYWORDS: Malnutrition; Hospitalized children.

\author{
${ }^{1}$ Médico residente del servicio de Gas- \\ troenterología y Nutrición Pediátrica. \\ ${ }^{2}$ Médico adscrito al servicio de Gas- \\ troenterología y Nutrición Pediátrica. \\ ${ }^{3}$ Jefe del servicio de Gastroenterología \\ y Nutrición Pediátrica. \\ ${ }^{4}$ Jefe del Departamento de Gastroente- \\ rología y Nutrición Pediátrica. \\ ${ }^{5}$ Médico de servicio social, Universidad \\ Anáhuac.
}

Instituto Nacional de Pediatría, Ciudad de México.

Recibido: 2 de agosto 2017

Aceptado: 26 de febrero 2018

Correspondencia

Ericka Montijo Barrios

erickamontijo@yahoo.com

Este artículo debe citarse como

García-Rivera R, Montijo-Barrios E, Cervantes-Bustamante $R$, ZárateMondragón F, Cadena-León JF, ToroMonjaraz EM, Cázares-Méndez $M$, López-Ugalde M, Ramírez-Mayans JA, Roldán-Montijo M. Métodos de evaluación de desnutrición intrahospitalaria en niños. Acta Pediatr Mex. 2018;39(4): 338-351. 


\section{INTRODUCCIÓN}

En la mayor parte de los países, un alto porcentaje de los niños hospitalizados sufren malnutrición. La prevalencia de desnutrición intrahospitalaria, de acuerdo con estadísticas de la OPS (Organización Panamericana de Salud) va de 30 a $40 \%$, y puede ser mayor en países en vías de industrialización, como el nuestro. ${ }^{1}$

La malnutrición en los pacientes hospitalizados tiene una repercusión considerable en el pronóstico, duración de la hospitalización y en los costos. ${ }^{2}$

En la actualidad, se recomienda que los pacientes que ingresan a un hospital sean valorados para determinar el riesgo de malnutrición, pues se ha reportado que, incluso $85 \%$ de quienes se hospitalizan, pierden peso durante su estancia. ${ }^{3}$

El estado nutricional de los niños es un indicador de salud y bienestar individual y poblacional. ${ }^{3} \mathrm{Es}$ decisivo conocer el estatus de la atención hospitalaria porque está directamente relacionado con la estancia y los costos derivados de ésta. ${ }^{4}$

La Sociedad Europea de Nutrición Clínica y Metabolismo (ESPEN, por sus siglas en inglés), la Sociedad Americana de Nutrición Parenteral y Enteral (ASPEN, por sus siglas en inglés) y la Sociedad Europea de Gastroenterología, Hepatología y Nutrición (ESPGHAN, por sus siglas en inglés) recomiendan la aplicación de herramientas de cribado para determinar el nivel de riesgo nutricional en pacientes pediátricos hospitalizados. ${ }^{1}$ La aplicación de estas herramientas facilita la detección temprana del deterioro nutricional y permite la planeación adecuada de la intervención nutricional. ${ }^{1}$ Por esto, las herramientas de cribado nutricional deben ser el primer paso en el diseño del plan nutricional durante la admisión y estancia hospitalaria. La OMS describe estas herramientas como una prueba simple para la identificación de individuos asintomáticos pero con algún padecimiento. ESPEN, por su parte, las define como un proceso rápido y simple efectuado por el equipo de admisión hospitalaria para identificar a los individuos con riesgo de desnutrición. $^{5}$

En el mundo se han diseñado diversas herramientas de cribado que ayudan a la prevención, identificación y manejo nutricional adecuado de la desnutrición hospitalaria infantil. Las principales herramientas enfocadas a la población infantil surgieron en los países industrializados y se describen en el Cuadro 1.6,7

Cuadro 1. Principales herramientas de cribado

\begin{tabular}{l|c|}
\hline $\begin{array}{l}\text { Herramienta de cribado } \\
\text { Nutritional Risk Score } \\
\text { (NRS) Tool }\end{array}$ & $\begin{array}{c}\text { Pacientes pediátricos y adultos } \\
(0-17 \text { años), sin cirugía. }\end{array}$ \\
$\begin{array}{l}\text { Simple Pediatric Nutri- } \\
\text { tional Risk Score (SPNRS) }\end{array}$ & $\begin{array}{c}\text { Pacientes pediátricos mayores } \\
\text { de } 1 \text { mes de edad a } \\
18 \text { años con y sin cirugía. }\end{array}$ \\
\hline $\begin{array}{l}\text { Subjective Global } \\
\text { Nutritional Assessmet for }\end{array}$ & $\begin{array}{c}\text { Pacientes pediátricos de 31 } \\
\text { días a } 17.9 \text { años de edad } \\
\text { Children (SGNA) }\end{array}$ \\
\hline $\begin{array}{l}\text { Screening Tool for As- } \\
\text { sessment of Malnutrition } \\
\text { in Paediatrics (STAMP) }\end{array}$ & $\begin{array}{c}\text { Pacientes pediátricos de 2 a } \\
17 \text { años de edad con y sin } \\
\text { cirugía. }\end{array}$ \\
$\begin{array}{l}\text { Screening Tool for Risk } \\
\text { On Nutritional status and } \\
\text { Growth (STRONGkids) }\end{array}$ & $\begin{array}{c}\text { Pacientes pediátricos de 1 } \\
\text { mes a } 16 \text { años de edad } \\
\text { con y sin cirugía. }\end{array}$ \\
$\begin{array}{l}\text { Paediatric Yorkhill Mal- } \\
\text { nutrition Score (PYMS) }\end{array}$ & $\begin{array}{c}\text { Pacientes pediátricos de 1 } \\
\text { a } 16 \text { años de edad } \\
\text { con y sin cirugía. }\end{array}$ \\
\hline
\end{tabular}

Tomado de: Moeeni V, Day AS. Nutritional risk screening tools in hospitalized children. Int J Child Health Nutr. 2012;(1):39-43.

El objetivo de este estudio fue: describir las distintas herramientas de cribado para el diagnóstico de desnutrición intrahospitalaria y comparar sus diferencias.

\section{MATERIAL Y MÉTODO}

Estudio comparativo y retrospectivo de búsqueda bibliográfica en las bases de datos: Scientific 
Electronic Library Online (SciELO), Red Informática de Medicina Avanzada (RIMA), Plataforma HIGHWIRE, Literatura Latinoamericana y del Caribe en Ciencias de la Salud (LILACS) y la Biblioteca Nacional de Medicina de Estados Unidos (Medline). Palabras clave de búsqueda (MeSH): herramientas de cribado nutricional, desnutrición intrahospitalaria y diagnóstico de malnutrición intrahospitalaria. Puesto que se trata de una revisión bibliográfica, y que las herramientas de cribado, aplicadas en distintos países, no se han validado se tomaron en cuenta todos los artículos arrojados por la búsqueda.

\section{RESULTADOS}

Descripción de las principales herramientas de cribado en población infantil

\section{Nutritional Risk Score (NRS) Tool ${ }^{8}$}

La desarrollaron Reilly y su grupo e incorporaron parámetros usados habitualmente:

1. Pérdida de peso (cantidad y duración).

2. Curvas de crecimiento (percentiles).

3. Ingesta dietética: apetito y habilidad para alimentarse y retención de alimento.

4. Factores de estrés: efectos de o las condiciones médicas en los requerimientos nutricionales.

Consta de dos fases: la inicial con cuatro preguntas. Si una es afirmativa debe continuarse con la fase final, en donde se da un puntaje con base en el que se decide dar o no apoyo nutricio. Si bien se ha aplicado en niños, su uso más extendido es en adultos. La primera fase es muy sencilla de aplicar por personal no médico.

\section{Simple Pediatric Nutritional Risk Score (SPNRS) ${ }^{9}$}

Fue desarrollada por Selmet-Gaudelus y sus colaboradores. Consiste en una evaluación com- pleta del riesgo nutricional en las primeras 48 horas del ingreso al hospital. Incluye entrevistas a los padres, personal de enfermería y, cuando es posible, al paciente hospitalizado. Los factores de riesgo nutricional señalados son:

1. Ingesta alimentaria: durante el proceso de admisión prescripción del tipo de alimentación apropiada para cada paciente según su padecimiento. Los padres, el personal de enfermería y nutrición evalúan el consumo diario durante las primeras 48 horas de estancia hospitalaria. Un investigador registra si la ingesta de alimentos fue de más de $50 \%$ o menos.

2. Dificultad para retener alimentos: se evalúa diariamente a través de la ocurrencia y número de episodios de vómito, diarrea o ambos.

3. Dolor: se evalúa con métodos apropiados para la edad. En el caso de los infantes, a los padres y al personal de enfermería se les pregunta si han observado signos de dolor, incluido Ilanto incesante, movimientos anormales y otras conductas que sugieran dolor. En el caso de los niños mayores de seis años se utiliza una escala analógica visual con puntuaciones desde 0 (sin dolor) hasta 100 (dolor intenso). El punto de corte para clasificar a un niño con dolor fue una puntuación mayor de 40.

4. Habilidad para alimentarse: se evalúa con base en criterios específicos, como: disfagia y alimentación asistida o a través de la observación de síntomas que interfieran con la ingestión de alimentos, como la disnea y la depresión. Categorizar si el paciente está "habilitado para alimentarse sin asistencia" o "requiere asistencia para la alimentación".

5. Condición patológica: se establece con tres categorías: media (grado 1), moderada (grado 2) y grave (grado 3). No había un sistema de clasificación válido para este rubro. 
a. Grado 1: toma en cuenta factores de estrés medio; por ejemplo, los procedimientos diagnósticos llevados a cabo en la admisión hospitalaria, infecciones menores que no requirieran hospitalización, otras enfermedades episódicas o cirugías menores.

b. Grado 2: coexistencia de factores de estrés moderado; por ejemplo, infecciones graves que no ponen en riesgo la vida del paciente, cirugía de rutina, fracturas, enfermedades agudas o enfermedad inflamatoria intestinal.

c. Grado 3: factores severos de estrés; por ejemplo, SIDA, neoplasias malignas, sepsis grave, cirugía mayor, múltiples lesiones, deterioro agudo por enfermedad crónica y depresión mayor.

El criterio final para la clasificación del riesgo nutricional fue el porcentaje de pérdida de peso corporal.

Con esta herramienta se identifican tres tipos de riesgo (Cuadro 2).

Una vez determinado el grado de deterioro nutricional se elige la intervención nutricional requerida para cada caso, como se muestra en el Cuadro 3.

Cuadro 2. Riesgo nutricional según SPNRS

\begin{tabular}{l|c|c|}
\hline Categoría & Puntaje & \multicolumn{1}{c}{ Criterios } \\
\hline $\begin{array}{l}\text { Pérdida nutricional } \\
\text { de bajo riesgo }\end{array}$ & 0 & \\
Pérdida nutricional & $1-2$ & $\begin{array}{c}\text { Condición patológica } \\
\text { grado } 2\end{array}$ \\
$\begin{array}{l}\text { de moderado riesgo } \\
\text { Pérdida nutricional } \\
\text { de alto riesgo }\end{array}$ & $\geq 3$ & $\begin{array}{c}\text { Condición patológica }<50 \% \\
\text { grado } 3\end{array}$ \\
\hline
\end{tabular}

Tomado de: Sermet-Gaudelus I, Poisson-Salomon AS, Colomb V, Brusset MC, Mosser F, Berrier F, et al. Simple pediatric nutritional risk score to identify children at risk of malnutrition. Am J Clin Nutr. 2000;72(1):64-70.

\section{Subjective Global Nutritional Assessment for} Children (SGNA) ${ }^{10}$

Fue adaptado y desarrollado por Secker y colaboradores. Los evaluadores, mediante una entrevista, obtienen información acerca de:

1. Peso y talla actuales y recientes del paciente.

2. Peso y talla actuales y recientes de los padres.

3. Ingesta alimentaria: tipo, volumen y frecuencia de líquidos y sólidos para infantes; frecuencia de ingestión de alimentos y una breve descripción del consumo diario; calificación de apetito y cambios recientes en el mismo; problemas de alimentación; restricciones en la dieta.

4. Frecuencia y duración de síntomas gastrointestinales: pérdida de apetito, vómito, diarrea y constipación para todas las edades y, además, dolor de estómago y náusea en el caso de los niños.

5. Capacidad funcional actual y cambios recientes: estado de alerta, cantidad de energía o nivel de actividad para infantes; asistencia escolar, habilidad para correr, jugar y practicar deportes con amigos $y$ tiempo de sueño.

6. Examen físico: signos físicos de pérdida de masa grasa y muscular, así como edema.

7. Presencia o ausencia de características históricas específicas, demandas metabólicas de la enfermedad subyacente, y signos físicos vinculados con la desnutrición.

Se consideran tres tipos de riesgo:

1. Estado de nutrición normal.

2. Desnutrición moderada.

3. Desnutrición grave.

Esta clasificación no utiliza algún sistema de puntuación con criterios específicos. 
Cuadro 3. Intervención nutricional según el grado de riesgo de malnutrición

\begin{tabular}{|c|c|c|c|c|}
\hline \multicolumn{2}{|c|}{ Factores de riesgo } & \multirow[b]{2}{*}{ Puntuación } & \multirow[b]{2}{*}{ Riesgo nutricional } & \multirow[b]{2}{*}{ Intervención nutricional } \\
\hline Patología & $\begin{array}{c}\text { Dolor [1] } \\
\text { Ingesta alimentaria } \\
<50 \%[1]\end{array}$ & & & \\
\hline Medio (grado 1) [0] & Ninguno & 0 & Bajo & Ninguno \\
\hline Medio (grado 1) [0] & Uno & 1 & Moderado & $\begin{array}{c}\text { Evaluación diaria de la ingesta } \\
\text { alimentaria y peso }\end{array}$ \\
\hline Medio (grado 1) [0] & Ambos & 2 & Moderado & Interconsulta con nutricionista \\
\hline Moderado (grado 2) [1] & Ninguno & 1 & Moderado & Inicio de soporte nutricional oral \\
\hline Moderado (grado 2) [1] & Uno & 2 & Moderado & \\
\hline Moderado (grado 2) [1] & Ambos & 3 & Alto & $\begin{array}{c}\text { Medición precisa de la ingesta } \\
\text { alimentaria }\end{array}$ \\
\hline Severo (grado 3) [3] & Ninguno & 3 & Alto & $\begin{array}{l}\text { Interconsulta con el equipo de } \\
\text { nutrición }\end{array}$ \\
\hline Severo (grado 3) [3] & Uno & 4 & Alto & $\begin{array}{l}\text { Consideración de introducir } \\
\text { nutrición enteral o parenteral }\end{array}$ \\
\hline Severo (grado 3) [3] & Ambos & 5 & Alto & \\
\hline
\end{tabular}

Tomado de: Sermet-Gaudelus I, Poisson-Salomon AS, Colomb V, Brusset MC, Mosser F, Berrier F, et al. Simple pediatric nutritional risk score to identify children at risk of malnutrition. Am J Clin Nutr 2000;72(1):64-70.

\section{Screening Tool for Assessment of Malnutrition in Paediatrics (STAMP) ${ }^{11}$}

Lo desarrollaron McCarthy y sus colaboradores. Considera cinco pasos; tres de ellos son elementos diagnósticos:

1. Diagnóstico: determina si el padecimiento tiene implicaciones nutricionales, como se refiere en el Cuadro 4.

2. Ingesta nutricional: dtermina cómo es ésta, como se refiere en el Cuadro 5.

3. Medición del peso y la talla como se reporta en el Cuadro 6.

4. Riesgo global de desnutrición como se menciona en el Cuadro 7.

5. Plan de cuidado, como se apunta en el

\section{Cuadro 8.}

Cuadro 4. Puntaje para el diagnóstico nutricional

\begin{tabular}{|l|c|c|}
\hline Criterio & Puntuación & Ejemplo criterio \\
\hline Definitivo & 3 & Disfagia \\
\hline Posible & 2 & $\begin{array}{c}\text { Problemas conductuales } \\
\text { de la alimentación }\end{array}$ \\
\hline No & 0 & Cirugía ambulatoria \\
\hline
\end{tabular}

Tomado de: McCarthy H, McNulty H, Dixon M, Eaton-Evans MJ. Screening for nutrition risk in children: the validation of a new tool. Hum Nutr Diet J 2008;21(4):395-96.

Cuadro 5. Puntaje para la ingesta nutricional

\begin{tabular}{|c|c|}
\hline Criterio & Puntuación \\
\hline Sin ingesta & 3 \\
\hline Ingesta disminuida recientemente-pobre & 2 \\
\hline Sin cambios-buena & 0
\end{tabular}

Tomado de: McCarthy H, McNulty H, Dixon M, Eaton-Evans MJ. Screening for nutrition risk in children: the validation of a new tool. Hum Nutr Diet J 2008;21(4):395-96. 
Cuadro 6. Puntaje para peso y talla

\section{Criterio}

Puntuación

$>3$ percentiles $/ \geq 3$ columnas por debajo del percentil 50

$>2$ percentiles $/=2$ columnas por debajo del percentil 50

0-1 percentil o columnas por debajo del percentil 50

McCarthy H, McNulty H, Dixon M, Eaton-Evans MJ. Screening for nutrition risk in children: the validation of a new tool. Hum Nutr Diet J 2008;21(4):395-96.

Cuadro 7. Puntaje para el riesgo de desnutrición

\begin{tabular}{|l|l|}
\hline Criterio & Puntuación \\
\hline Alto riesgo & $\geq 4$ \\
\hline Medio riesgo & $2-3$ \\
\hline Bajo riesgo & $0-1$ \\
\hline
\end{tabular}

Tomado de: McCarthy H, McNulty H, Dixon M, Eaton-Evans MJ. Screening for nutrition risk in children: the validation of a new tool. Hum Nutr Diet J 2008;21(4):395-96.

Cuadro 8. Plan de tratamiento según la puntuación global

\begin{tabular}{|c|c|}
\hline Criterio & Puntuación \\
\hline Alto riesgo & $\begin{array}{l}\text { - Interconsulta con el equipo de soporte } \\
\text { nutricional } \\
\text { - Monitoreo según el plan de cuidado }\end{array}$ \\
\hline $\begin{array}{l}\text { Mediano } \\
\text { riesgo }\end{array}$ & $\begin{array}{l}\text { - Monitoreo de la ingesta nutricional } \\
\text { durante } 3 \text { días } \\
\text { - Repetición de la STAMP después de } 3 \\
\text { días } \\
\text { - Reajustes al plan de cuidado según se } \\
\text { requiera }\end{array}$ \\
\hline Bajo riesgo & $\begin{array}{l}\text { - Continuación del plan de cuidados } \\
\text { clínicos rutinarios } \\
\text { - Repetición semanal de la STAMP durante } \\
\text { la estancia hospitalaria } \\
\text { - Reajustes al plan de cuidado según se } \\
\text { requiera }\end{array}$ \\
\hline
\end{tabular}

Tomado de: McCarthy H, McNulty H, Dixon M, Eaton-Evans MJ. Screening for nutrition risk in children: the validation of a new tool. Hum Nutr Diet J 2008;21(4):395-96.
Screening Tool for Risk On Nutritional status and Growth (STRONGkids) ${ }^{12}$

Lo desarrollaron Hust y su grupo. Consiste en cuatro ítems y cada uno tiene una puntuación entre 1-2 con máxima de 5. Incluye:

1. Evaluación clínica subjetiva (1 punto): se evalúa subjetivamente si hay disminución de masa grasa subcutánea y muscular o rostro hundido.

2. Enfermedad de alto riesgo (2 puntos): se evalúa si hay alguna enfermedad subyacente que predisponga a riesgo de desnutrición o cirugía mayor:

- Displasia broncopulmonar

- Enfermedad celiaca

- Fibrosis quística

- Enfermedad cardiaca (se evalúa cronicidad)

- Enfermedad infecciosa (SIDA)

- Enfermedad inflamatoria intestinal

- Cáncer

- Enfermedad hepática (se evalúa cronicidad)

- Enfermedad renal (se evalúa cronicidad)

- Pancreatitis

- Síndrome de intestino corto

- Enfermedad muscular

- Enfermedad metabólica

- Traumatismo

- Anorexia nerviosa

- Quemaduras

- Prematurez

- Discapacidad-retraso mental

- Cirugía mayor 
3. Ingesta nutricional y pérdidas (1 punto): se evalúa si hay:

- Diarrea excesiva ( $\geq 5$ veces al día) o vómito (más de 3 veces aldía) en los últimos días.

- Reducción de la ingestión durante los últimos días antes del ingreso al hospital.

- Preexistencia de un plan nutricional.

4. Pérdida de peso o pobre ganancia de peso (infantes menores de 1 año) durante las últimas semanas o meses.

Para evaluar el riesgo de desnutrición se consideran tres categorías: (Cuadro 9)

Cuadro 9. Categorías para el riesgo nutricional

\begin{tabular}{|l|c|}
\hline Criterio & Puntuación \\
\hline Alto riesgo & $4-5$ \\
\hline Moderado riesgo & $1-3$ \\
\hline Bajo riesgo & 0 \\
\hline
\end{tabular}

Tomado de: Hulst JM, Zwart H, Hop EC, Joosten K FM. Dutch national survey to test the STRONG kids nutritional risk screening tool in hospitalized children. Clin Nutr 2010;29(1):106-111.

\section{Pediatric Yorkhill Malnutrition Score (PYMS) ${ }^{13}$}

Desarrollada por Gerasmidis y sus coautores, la evaluación consta de cinco pasos y tiene un puntaje máximo de 7 :

1. Puntaje del índice de masa corporal: el valor obtenido se compara con los puntos de corte.
a. Por debajo $=\mathrm{NO}=0$ puntos.
b. Por debajo $=$ SÍ $=2$ puntos.

2. Pérdida de peso: se evalúa la pérdida de peso no intencional y pobre ganancia de peso si el niño es menor de dos años. $\mathrm{Si}$ cualquiera de las dos condiciones anteriores se cumple, se asigna un valor de 1 ; si no, obtiene 0 .

3. Evaluación de cambios recientes en la dieta o soporte nutricional:

a. Ingestión sin cambios $=\mathrm{NO}=0$ puntos.

b. Consumo acostumbrado disminuido al menos en la semana pasada $=$ SÍ = 1 punto.

c. Sin ingesta (o sólo pequeñas porciones líquidas) al menos en la semana pasa$\mathrm{da}=\mathrm{S} I ́=2$ puntos

4. Efectos del ingreso hospitalario sobre la condición del paciente:

a. Sin cambios $=0$ puntos.

b. En la última semana de estancia hospitalaria se ha disminuido la ingesta o se han incrementado los requerimientos o se han incrementado las pérdidas $=$ sí = 1 punto.

c. En la última semana de estancia hospitalaria no ha habido ingesta $=$ Sí $=$ 2 puntos.

5. Puntaje total y plan de acción:

a. Puntaje total mayor de 2 refleja un riesgo nutricional significativo.

b. Puntaje total $=2$ requiere revisión dietética.

c. Puntaje total $=1$ refleja un buen balance y la ingesta debe monitorearse cuidadosamente. Se considera repetir la evaluación al tercer día.

d. Puntaje total $=0$ repetir la prueba cada semana.

Esta evaluación debe completarse antes de que transcurran 24 horas de la admisión. 


\section{DISCUSIÓN}

Cada herramienta busca evaluar subjetiva y objetivamente el riesgo de pérdida de peso del niño hospitalizado. Algunas, como NRS, se aplicaron primero en adultos y después en niños. En general, todas las escalas recaban datos de la forma de alimentación, pérdida de peso, gravedad de la enfermedad, entre otros, que son de tipo subjetivo, y algunas usan datos objetivos de antropometría y de laboratorio. En el Cuadro 10 se describen las herramientas, su diseño y las escalas de medición que utilizan. ${ }^{6,7}$

Todas estas herramientas pretenden orientar la conducta del personal médico para que la intervención dietética sea oportuna. Enseguida se enlistan las acciones predefinidas de acuerdo con el resultado del cribaje nutricional aplicado en el Cuadro 11.

En el Cuadro 12 se hace un comparativo de la sensibilidad y especificidad de las herramientas de cribado y un patrón de referencia (la mejor herramienta de cribado nutricional). Solo Wonoputri y su grupo ${ }^{1}$ hicieron esta comparación en la que tomaron como referencia a la SGNA. En ese estudio se menciona que la herramienta más confiable es la PYMS. Los otros porcentajes mostrados en el cuadro corresponden a los datos reportados por los autores de las pruebas.

En el Cuadro 13 se observa la gran variabilidad de resultados cuando las herramientas se comparan entre sí. Lo anterior refleja la falta de consenso acerca de la forma óptima de evaluar el riesgo nutricional en niños y la falta de estandarización para la aplicación de las herramientas (por ejemplo, enfermeras vs nutricionistas).

Se ha demostrado ${ }^{14}$ que algunas de las herramientas de cribado nutricional pueden detectar con precisión y exactitud el riesgo nutricional en los niños; sin embargo, no hay evidencia que sustente que estas pruebas mejoren los pronósticos y disminuyan el tiempo de hospitalización. Hace poco se sugirió que la investigación futura debería centrarse en demostrar cómo es que las herramientas de cribado mejoran los resultados en niños con y sin riesgo nutricional.

En nuestra institución Pinilla y colaboradores ${ }^{15}$ efectuaron un estudio en el que se reportó que sólo en $43 \%$ de los pacientes hospitalizados se analizan los indicadores de malnutrición, como: peso para la edad, peso para la talla y talla para la edad, y más grave aún sólo en $24 \%$ se estableció un diagnóstico nutricional. En 84\% de los pacientes no hubo alguna intervención dietética al ingreso, aun cuando se trató de niños con desnutrición moderada, grave y obesidad. Así mismo, se observó que gran número de pacientes perdió peso durante la hospitalización; sin embargo, es un dato difícil de determinar, pues a $40.4 \%$ de los pacientes no se les pesó al momento de su egreso.

La guía de CENETEC ${ }^{2}$ de desnutrición intrahospitalaria: tamizaje diagnóstico y tratamiento anota que la atención nutricional es un derecho y debe recibirla todo paciente que ingresa a una institución hospitalaria, por lo que resulta obligatorio que se otorgue a todo paciente con desnutrición o en riesgo de padecerla; así mismo, indica que a todos los pacientes hospitalizados se les debe hacer una evaluación nutricional integral que proporcione datos de los antecedentes de importancia y padecimientos actuales para la obtención del cálculo adecuado de sus requerimientos nutricionales.

Esta guía sugiere métodos de tamizaje de riesgo de desnutrición intrahospitalaria:

- Parámetros antropométricos: peso, IMC y pérdida ponderal.

- Ingesta dietética y comportamiento con el peso. 
Cuadro 10. Descripción de las principales herramientas de cribado (continúa en la siguiente página)

\begin{tabular}{|c|c|c|c|c|c|}
\hline $\begin{array}{l}\text { Herramienta-país- } \\
\text { año-lugar }\end{array}$ & Diseño & $\begin{array}{l}\text { Temporalidad- } \\
\text { características de } \\
\text { la población }\end{array}$ & $\begin{array}{l}\text { Criterios de } \\
\text { inclusión- } \\
\text { exclusión }\end{array}$ & Componentes & $\begin{array}{l}\text { Escala de medición- } \\
\text { comentarios }\end{array}$ \\
\hline $\begin{array}{l}\text { - Nutritional Risk Score } \\
\text { (NRS) Tool } \\
\text { - Birmingham Heart } \\
\text { lands Hospital } \\
\text { - Reino Unido }\end{array}$ & Prospectivo & $\begin{array}{l}\text { - Un hospital } \\
\text { - } 150 \text { niños }\end{array}$ & & $\begin{array}{l}\text { - Técnicas básicas } \\
\text { de evaluación } \\
\text { nutricional } \\
\text { (peso, talla) } \\
\text { - Ingesta de } \\
\text { alimentos }\end{array}$ & $\begin{array}{l}\text { Inicialmente se } \\
\text { desarrolló en } \\
\text { población adulta } \\
\text { Se asignaron tres } \\
\text { categorías: bajo, } \\
\text { moderado y alto } \\
\text { riesgo }\end{array}$ \\
\hline $\begin{array}{l}\text { - Simple pediatric } \\
\text { nutritional risk score } \\
(\text { SPNRS })^{8} \\
\text { - Francia } \\
\text { - } 1997 \\
\text { - Necker-Enfants Mala- } \\
\text { dies Hospital }\end{array}$ & Prospectivo & $\begin{array}{l}\text { - Marzo-mayo } 1997 \\
\text { - } 96 \text { niños admi- } \\
\text { tidos consecuti- } \\
\text { vamente en alguna } \\
\text { especialidad } \\
\text { médica (gastroen- } \\
\text { terología, cardio- } \\
\text { logía, neumolo- } \\
\text { gía, hematología o } \\
\text { pediatría general) } \\
\text { o cirugía }\end{array}$ & $\begin{array}{l}\text { Inclusión: } \\
\text { estancia en el } \\
\text { hospital }>48 \\
\text { horas y edad >1 } \\
\text { mes } \\
\text { Exclusión: } \\
\text { niños con } \\
\text { implicaciones } \\
\text { que condiciona- } \\
\text { ban variaciones } \\
\text { importantes de } \\
\text { la hidratación } \\
\text { (hepatopatía, } \\
\text { nefropatía o } \\
\text { insuficiencia } \\
\text { cardiaca severa) }\end{array}$ & $\begin{array}{l}\text { - Ingesta de ali- } \\
\text { mentos } \\
\text { - Dificultades para } \\
\text { la retención de } \\
\text { alimentos (dia- } \\
\text { rrea y vómito) } \\
\text { - Dolor } \\
\text { - Habilidades para } \\
\text { alimentarse }\end{array}$ & $\begin{array}{l}\text { Se consideró para } \\
\text { clasificar a un niño } \\
\text { con riesgo } \\
\text { nutricional, una } \\
\text { pérdida de peso } \\
\text { mayor de } 2 \%\end{array}$ \\
\hline $\begin{array}{l}\text { - Subjective Global Nu } \\
\text { tritional Assessmet for } \\
\text { Children (SGNA) } \\
\text { - Canadá } \\
\text { - The Hospital for Sick } \\
\text { Children }\end{array}$ & Prospectivo & $\begin{array}{l}\text { - Febrero } \\
\text { 2003-agosto } 2004 \\
\text { - } 175 \text { niños }\end{array}$ & $\begin{array}{l}\text { Inclusión: niños } \\
\text { de } 31 \text { días a } \\
17.9 \text { años de } \\
\text { edad que requi- } \\
\text { rieran cirugía } \\
\text { mayor abdomi- } \\
\text { nal o torácica } \\
\text { no cardiaca no } \\
\text { urgente y que no } \\
\text { hubiesen sido } \\
\text { intervenidos qui- } \\
\text { rúrgicamente } 30 \\
\text { días antes de la } \\
\text { aplicación de la } \\
\text { herramienta } \\
\text { Exclusión: niños } \\
\text { nacidos a pretér- } \\
\text { mino, clínica- } \\
\text { mente inestables } \\
\text { y que ellos y sus } \\
\text { cuidadores no } \\
\text { hablaran inglés }\end{array}$ & $\begin{array}{l}\text { - Peso y talla } \\
\text { histórico } \\
\text { - Ingesta dietética } \\
\text { - Frecuencia y du- } \\
\text { ración de sínto- } \\
\text { mas gastrointes- } \\
\text { tinales } \\
\text { - Capacidad fun- } \\
\text { cional actual } \\
\text { - Examen } \\
\text { físico } \\
\text { - Presencia o } \\
\text { ausencia de } \\
\text { características } \\
\text { históricas espe- } \\
\text { cíficas } \\
\text { - Demandas } \\
\text { metabólicas de } \\
\text { la enfermedad } \\
\text { subyacente } \\
\text { - Signos físicos } \\
\text { asociados con } \\
\text { desnutrición }\end{array}$ & $\begin{array}{l}\text { Los niños se } \\
\text { clasificaron según } \\
\text { las complicaciones } \\
\text { infecciosas y no in- } \\
\text { fecciosas; duración } \\
\text { de la hospitaliza- } \\
\text { ción posoperatoria, } \\
\text { tratamiento con } \\
\text { antibióticos no } \\
\text { profilácticos y las } \\
\text { tasas de readmisión } \\
\text { no planeadas } \\
\text { Contiene elemen- } \\
\text { tos subjetivos y } \\
\text { objetivos } \\
\text { Se asignaron tres } \\
\text { categorías: normal, } \\
\text { moderado y severo }\end{array}$ \\
\hline
\end{tabular}


Cuadro 10. Descripción de las principales herramientas de cribado (continúa en la siguiente página)

\begin{tabular}{|c|c|c|c|c|c|}
\hline $\begin{array}{l}\text { Herramienta-país- } \\
\text { año-lugar }\end{array}$ & Diseño & $\begin{array}{l}\text { Temporalidad- } \\
\text { características de } \\
\text { la población }\end{array}$ & $\begin{array}{l}\text { Criterios de } \\
\text { inclusión- } \\
\text { exclusión }\end{array}$ & Componentes & $\begin{array}{l}\text { Escala de medición- } \\
\text { comentarios }\end{array}$ \\
\hline $\begin{array}{l}\text { Screening Tool for As- } \\
\text { sessment of Malnu- } \\
\text { trition in Paediatrics } \\
\left(\text { STAMP) }{ }^{10}\right. \\
\text { - Reino Unido } \\
\text { - } 2008\end{array}$ & & $\begin{array}{l}89 \text { niños admitidos } \\
\text { en salas médicas o } \\
\text { de cirugía }\end{array}$ & & $\begin{array}{l}\text { - Diagnóstico } \\
\text { clínico y sus } \\
\text { implicaciones } \\
\text { nutricionales } \\
\text { - Ingesta dietética } \\
\text { - Medidas antro- } \\
\text { pométricas }\end{array}$ & $\begin{array}{l}\text { Se asignaron tres } \\
\text { categorías: bajo, } \\
\text { medio y alto riesgo }\end{array}$ \\
\hline $\begin{array}{l}\text { - Screening Tool for Risk } \\
\text { On Nutritional status } \\
\text { and Growth } \\
\text { (STRONGkids) }^{11} \\
\text { - Holanda } \\
\text { - } 2007\end{array}$ & $\begin{array}{l}\text { Multi- } \\
\text { céntrico, } \\
\text { prospectivo } \\
\text { y observa- } \\
\text { cional }\end{array}$ & $\begin{array}{l}\text { - Noviembre } 2007 \\
\text { - } 44 \text { hospitales } \\
\text { - } 424 \text { niños }\end{array}$ & $\begin{array}{l}\text { Inclusión: niños } \\
\text { mayores de } 1 \\
\text { mes y una estan- } \\
\text { cia hospitalaria } \\
\text { de al menos } \\
1 \text { día } \\
\text { Exclusión: pa- } \\
\text { cientes críticos }\end{array}$ & $\begin{array}{l}\text { - Evaluación } \\
\text { clínica subjetiva } \\
\text { (1 punto) } \\
\text { - Enfermedad } \\
\text { de alto riesgo (2 } \\
\text { puntos) } \\
\text { - Ingesta nutricio- } \\
\text { nal (1 punto) } \\
\text { - Pérdida de peso } \\
\text { o pobre ganancia } \\
\text { de peso (1 punto) }\end{array}$ & $\begin{array}{l}\text { Se clasificó como } \\
\text { riesgo nutricional } \\
\text { un puntaje igual o } \\
\text { mayor a } 4\end{array}$ \\
\hline 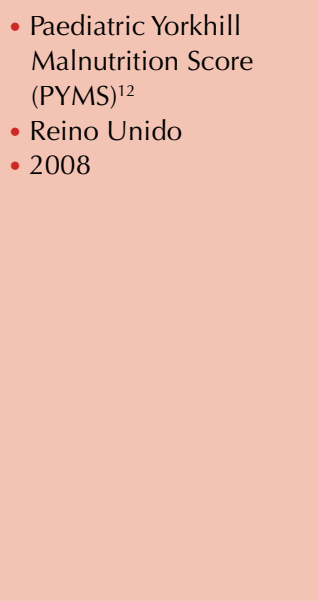 & $\begin{array}{l}\text { Estudio } \\
\text { piloto de } \\
\text { validación }\end{array}$ & $\begin{array}{l}\text { - Junio-octubre } \\
2008 \\
\text { - Cuatro hospitales } \\
\text { - } 247 \text { niños }\end{array}$ & & $\begin{array}{l}\text { - Estado nutri- } \\
\text { cional de los } \\
\text { pacientes evalua- } \\
\text { dos con el índice } \\
\text { de masa corporal } \\
\text { (0-2 puntos) } \\
\text { - Cambios recien- } \\
\text { tes en el estado } \\
\text { nutricional (0-2 } \\
\text { puntos) } \\
\text { - Posibilidad } \\
\text { de deterioro del } \\
\text { estado nutricio- } \\
\text { nal como resulta- } \\
\text { do de la patolo- } \\
\text { gía en curso (0-2 } \\
\text { puntos) }\end{array}$ & $\begin{array}{l}\text { Se realizaron tres } \\
\text { clasificaciones: } \\
\text { bajo (0 puntos), } \\
\text { medio y alto riesgo } \\
\text { nutricional ( } 1 \text { o } 2 \\
\text { puntos, respectiva- } \\
\text { mente) }\end{array}$ \\
\hline
\end{tabular}

- Diseñar una herramienta validada para la población donde se va a aplicar.

- Aplicar la escala de valoración mínima nutricional (MNA).

- Aplicar el NRS 2002.

\section{CONCLUSIONES}

Durante el curso de la estancia intrahospitalaria el estado nutricional se va progresivamente energética por enfermedad o un evento agudo agregado. Debido a la existencia de múltiples variables ni en Latinoamérica ni en nuestro país existe una herramienta validada como patrón de referencia del estado nutricional; esto es la causa de la diversidad en los resultados. Por esto se sugiere el desarrollo de una herramienta de fácil aplicación y resultados confiables.

\section{REFERENCIAS}

1. Wonoputri N, Djais JT, Rosalina I. Validity of nutritional screening tools for hospitalized children. J Nutr Metab. 2014;2014:1436-49. 
Cuadro 11. Acciones predefinidas según el resultado del cribaje nutricional

\begin{tabular}{|c|c|c|c|}
\hline & Riesgo bajo & Riesgo moderado & Riesgo alto \\
\hline $\begin{array}{l}\text { Nutritional Risk Score (NRS) } \\
\text { Tool }\end{array}$ & $\begin{array}{l}\text { No hay acciones } \\
\text { predefinidas }\end{array}$ & $\begin{array}{l}\text { No hay acciones } \\
\text { predefinidas }\end{array}$ & $\begin{array}{l}\text { No hay acciones } \\
\text { predefinidas }\end{array}$ \\
\hline $\begin{array}{l}\text { Simple Pediatric Nutritional } \\
\text { Risk Score (SPNRS) }\end{array}$ & No aplica & $\begin{array}{l}\text { Vigilancia de peso, reportes } \\
\text { de ingesta de alimentos; } \\
\text { se evalúa la necesidad de } \\
\text { consulta nutricional }\end{array}$ & $\begin{array}{l}\text { Evaluación nutricional, moni- } \\
\text { toreo de ingesta de alimentos; } \\
\text { se evalúa la necesidad de } \\
\text { consulta nutricional }\end{array}$ \\
\hline $\begin{array}{l}\text { Screening Tool for Assess- } \\
\text { ment of Malnutrition in } \\
\text { Paediatrics (STAMP) }\end{array}$ & $\begin{array}{l}\text { Repetición de la herramien- } \\
\text { ta } 1 \text { semana posterior a la } \\
\text { primera evaluación }\end{array}$ & $\begin{array}{l}\text { Monitoreo de la ingesta de } \\
\text { alimentos por } 3 \text { días }\end{array}$ & Consulta de nutrición \\
\hline $\begin{array}{l}\text { Screening Tool for Risk On } \\
\text { Nutritional status and Growth } \\
\text { (STRONGkids) }\end{array}$ & $\begin{array}{l}\text { Repetición de la herramien- } \\
\text { ta } 1 \text { semana posterior a la } \\
\text { primera evaluación }\end{array}$ & $\begin{array}{l}\text { Revisión de peso } 2 \text { veces por } \\
\text { semana; se evalúa la necesi- } \\
\text { dad de consulta nutricional }\end{array}$ & Consulta de nutrición \\
\hline $\begin{array}{l}\text { Paediatric Yorkhill } \\
\text { Malnutrition Score (PYMS) }\end{array}$ & $\begin{array}{l}\text { Repetición de la herramien- } \\
\text { ta } 1 \text { semana posterior a la } \\
\text { primera evaluación }\end{array}$ & $\begin{array}{l}\text { Repetición de la herramienta } \\
3 \text { días posteriores a la primera } \\
\text { evaluación }\end{array}$ & $\begin{array}{l}\text { Consulta de nutrición; la } \\
\text { intervención nutricional es } \\
\text { importante }\end{array}$ \\
\hline
\end{tabular}

Cuadro 12. Comparativo de sensibilidad y especificidad de las diferentes herramientas de cribado

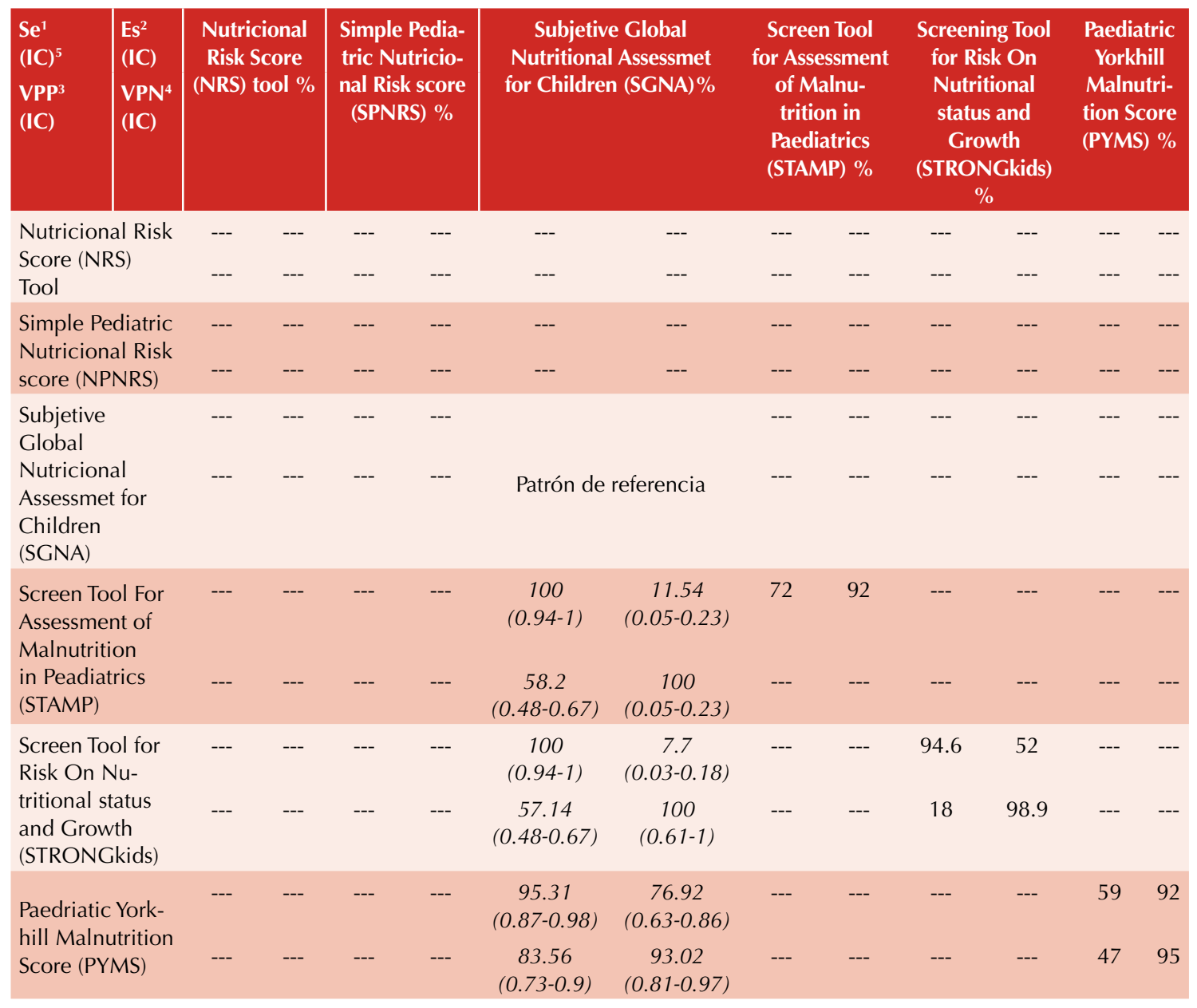


Cuadro 13. Comparativo de sensibilidad y especificidad entre herramientas de cribado nutricional para la detección de pérdidas de peso corporal mayores de $2^{14}$

\section{A1. Bajo riesgo vs moderado MÁS alto riesgo}

\begin{tabular}{l|c|c} 
Estudio & $\begin{array}{c}\text { Sensibilidad } \\
\text { (IC95\%) }\end{array}$ & $\begin{array}{c}\text { Especificidad } \\
(\text { IC95\%) }\end{array}$ \\
\hline $\begin{array}{l}\text { Simple Pediatric } \\
\text { Nutritional Risk Score } \\
\text { (SPNRS) }\end{array}$ & $\begin{array}{c}0.99 \\
(0.96,1.00)\end{array}$ & $\begin{array}{c}0.27 \\
(0.20,0.34)\end{array}$ \\
$\begin{array}{l}\text { Screening Tool for Risk } \\
\text { On Nutritional Status } \\
\text { and Growth }\end{array}$ & 0.61 & 0.36 \\
$\begin{array}{l}\text { (STRONGkids) } \\
\text { Screening Tool for Risk }\end{array}$ & $0.41,0.78)$ & $(0.30,0.43)$ \\
$\begin{array}{l}\text { On Nutritional Status } \\
\text { and Growth } \\
\text { (STRONGkids) }\end{array}$ & $(0.46,0.59)$ & $(0.34,0.53)$ \\
& & \\
\hline
\end{tabular}

Tomado de: Huysentruyt, K, Devreker T, Dejonchheer J, De Schepper J, Vandenplas I, Cool F. The Accuracy of Nutritional Screening Tools in Assessing the Risk of Undernutrition in Hospitalized Children: A Systematic Review of Literature and Meta-analysis. J Pediatr Gastroenterol Nutr 2015;61(2):159-66.

\section{A2. Bajo riesgo MÁS moderado vs alto riesgo}

\begin{tabular}{|c|c|c|}
\hline Estudio & $\begin{array}{l}\text { Sensibilidad } \\
\text { (IC95\%) }\end{array}$ & $\begin{array}{c}\text { Especificidad } \\
\text { (IC95\%) }\end{array}$ \\
\hline $\begin{array}{l}\text { Simple Pediatric } \\
\text { Nutritional Risk Score } \\
\text { (SPNRS) }\end{array}$ & $\begin{array}{c}0.76(0.68 \\
0.73)\end{array}$ & $\begin{array}{c}0.82(0.75 \\
0.88)\end{array}$ \\
\hline $\begin{array}{l}\text { Screening Tool for Risk } \\
\text { On Nutritional Status } \\
\text { and Growth } \\
\text { (STRONGkids) }\end{array}$ & $\begin{array}{c}0.00(0.00 \\
0.12)\end{array}$ & $\begin{array}{c}0.93(0.89 \\
0.96)\end{array}$ \\
\hline $\begin{array}{l}\text { Screening Tool for Risk } \\
\text { On Nutritional Status } \\
\text { and Growth } \\
\text { (STRONGkids) }\end{array}$ & $\begin{array}{c}0.06(0.03 \\
0.09)\end{array}$ & $\begin{array}{c}0.88(0.80 \\
0.93)\end{array}$ \\
\hline
\end{tabular}

Tomado de: Huysentruyt, K, Devreker T, Dejonchheer J, De Schepper J, Vandenplas I, Cool F. The Accuracy of Nutritional Screening Tools in Assessing the Risk of Undernutrition in Hospitalized Children: A Systematic Review of Literature and Meta-analysis. J Pediatr Gastroenterol Nutr 2015;61(2):159-66.
B1. Comparativo de sensibilidad y especificidad entre las herramientas de cribado nutricional para la valoración dietética referida ${ }^{14}$

\section{Bajo riesgo vs moderado MÁS alto riesgo}

\begin{tabular}{|c|c|c|}
\hline Estudio & $\begin{array}{l}\text { Sensibilidad } \\
\text { (IC95\%) }\end{array}$ & $\begin{array}{c}\text { Especificidad } \\
\text { (IC95\%) }\end{array}$ \\
\hline $\begin{array}{l}\text { Paediatric Yorhill } \\
\text { Malnutrition Score } \\
\text { (PYMS) }\end{array}$ & $\begin{array}{l}0.62 \quad(0.42, \\
0.79)\end{array}$ & $\begin{array}{l}0.66 \\
0.76)\end{array}$ \\
\hline $\begin{array}{l}\text { Screening Tool for } \\
\text { Assessment of } \\
\text { Malnutrition in } \\
\text { Paediatrics (STAMP) }\end{array}$ & $\begin{array}{l}0.97 \quad 0.82, \\
1.00)\end{array}$ & $\begin{array}{l}0.26 \quad 0.18 \\
0.74)\end{array}$ \\
\hline $\begin{array}{l}\text { Screening Tool for Risk } \\
\text { On Nutritional Status } \\
\text { and Growth } \\
\text { (STRONGkids) }\end{array}$ & $\begin{array}{l}0.93 \quad 0.77, \\
0.99)\end{array}$ & $\begin{array}{l}0.44 \quad 0.35 \\
0.52)\end{array}$ \\
\hline
\end{tabular}

Tomado de: Huysentruyt, K, Devreker T, Dejonchheer J, De Schepper J, Vandenplas I, Cool F. The Accuracy of Nutritional Screening Tools in Assessing the Risk of Undernutrition in Hospitalized Children: A Systematic Review of Literature and Meta-analysis. J Pediatr Gastroenterol Nutr 2015;61(2):159-66.

\section{B2. Bajo riesgo MÁS moderado vs alto riesgo}

\begin{tabular}{l|c|c|}
\hline Estudio & $\begin{array}{c}\text { Sensibilidad } \\
\text { (IC95\%) }\end{array}$ & $\begin{array}{c}\text { Especificidad } \\
\text { (IC95\%) }\end{array}$ \\
\hline $\begin{array}{l}\text { Paediatric Yorhill Mal- } \\
\text { nutrition Score (PYMS) }\end{array}$ & $\begin{array}{c}0.45(0.26, \\
0.64)\end{array}$ & $\begin{array}{c}0.80(0.72, \\
0.86)\end{array}$ \\
\hline $\begin{array}{l}\text { Screening Tool for As- } \\
\text { sessment of Malnutri- }\end{array}$ & $0.41(0.24$. & $0.76(0.68$, \\
tion in Paediatrics & $0.61)$ & $.083)$ \\
(STAMP) & & \\
Screening Tool for & $0.14(0.04$, & $0.95,1.00)$ \\
Risk On Nutritional & $0.32)$ & \\
Status and Growth & & \\
(STRONGkids) & &
\end{tabular}

Tomado de: Huysentruyt, K, Devreker T, Dejonchheer J, De Schepper J, Vandenplas I, Cool F. The Accuracy of Nutritional Screening Tools in Assessing the Risk of Undernutrition in Hospitalized Children: A Systematic Review of Literature and Meta-analysis. J Pediatr Gastroenterol Nutr 2015;61(2):159-66. 
C1. Comparativo de sensibilidad y especificidad entre las herramientas de cribado nutricional para la valoracion dietética directa ${ }^{14}$

\section{Bajo riesgo vs moderado MÁS alto riesgo}

\begin{tabular}{|c|c|c|}
\hline Estudio & $\begin{array}{l}\text { Sensibilidad } \\
\text { (IC95\%) }\end{array}$ & $\begin{array}{l}\text { Especificidad } \\
\text { (IC95\%) }\end{array}$ \\
\hline $\begin{array}{l}\text { Paediatric Yorhill Malnu- } \\
\text { trition Score (PYMS) }\end{array}$ & $\begin{array}{c}0.45 \\
(0.34,0.57)\end{array}$ & $\begin{array}{c}0.84 \\
(0.78,0.89)\end{array}$ \\
\hline $\begin{array}{l}\text { Screening Tool for As- } \\
\text { sessment of Malnutrition } \\
\text { in Paediatrics (STAMP) }\end{array}$ & $\begin{array}{c}0.96 \\
(0.24,0.61)\end{array}$ & $\begin{array}{c}0.45 \\
(0.38,0.53)\end{array}$ \\
\hline $\begin{array}{l}\text { Screening Tool for As- } \\
\text { sessment of Malnutrition } \\
\text { in Paediatrics (STAMP) }\end{array}$ & $\begin{array}{c}0.92(0.04 \\
0.32)\end{array}$ & $\begin{array}{c}0.23 \\
(0.17,0.29)\end{array}$ \\
\hline $\begin{array}{l}\text { Screening Tool for As- } \\
\text { sessment of Malnutrition } \\
\text { in Paediatrics (STAMP) }\end{array}$ & $\begin{array}{c}1.00 \\
(0.04,1.00)\end{array}$ & $\begin{array}{c}0.46 \\
(0.39,0.53)\end{array}$ \\
\hline
\end{tabular}

Tomado de: Huysentruyt, K, Devreker T, Dejonchheer J, De Schepper J, Vandenplas I, Cool F. The Accuracy of Nutritional Screening Tools in Assessing the Risk of Undernutrition in Hospitalized Children: A Systematic Review of Literature and Meta-analysis. J Pediatr Gastroenterol Nutr 2015;61(2):159-66

\section{C2. Bajo riesgo MÁS moderado vs alto riesgo}

\begin{tabular}{|c|c|c|}
\hline Estudio & $\begin{array}{l}\text { Sensibilidad } \\
\text { (IC95\%) }\end{array}$ & $\begin{array}{l}\text { Especificidad } \\
\text { (IC95\%) }\end{array}$ \\
\hline $\begin{array}{l}\text { Paediatric Yorhill Mal- } \\
\text { nutrition Score (PYMS) }\end{array}$ & $\begin{array}{l}0.5980 .39 \\
0.78)\end{array}$ & $\begin{array}{c}0.84(0.78, \\
0.89)\end{array}$ \\
\hline $\begin{array}{l}\text { Screening Tool for As- } \\
\text { sessment of Malnutri- } \\
\text { tion in Paediatrics } \\
\text { (STAMP) }\end{array}$ & $\begin{array}{c}0.81(0.62 \\
0.94)\end{array}$ & $\begin{array}{c}0.45(0.38 \\
0.53)\end{array}$ \\
\hline $\begin{array}{l}\text { Screening Tool for As- } \\
\text { sessment of Malnutri- } \\
\text { tion in Paediatrics } \\
\text { (STAMP) }\end{array}$ & $\begin{array}{c}0.75(0.63 \\
0.85)\end{array}$ & $\begin{array}{c}0.23(0.17 \\
0.29)\end{array}$ \\
\hline $\begin{array}{l}\text { Screening Tool for } \\
\text { Risk On Nutritional } \\
\text { Status and Growth } \\
\text { (STRONGkids) }\end{array}$ & $\begin{array}{l}0.70(0.51 \\
\quad 0.84)\end{array}$ & $\begin{array}{c}0.46(0.39 \\
0.53)\end{array}$ \\
\hline
\end{tabular}

Tomado de: Huysentruyt, K, Devreker T, Dejonchheer J, De Schepper J, Vandenplas I, Cool F. The Accuracy of Nutritional Screening Tools in Assessing the Risk of Undernutrition in Hospitalized Children: A Systematic Review of Literature and Meta-analysis. J Pediatr Gastroenterol Nutr 2015;61(2):159-66.
D1. Comparativo de sensibilidad y especificidad entre las herramientas de cribado nutricional para la intervención nutricional ${ }^{14}$

Bajo riesgo vs moderado MÁS alto riesgo

\begin{tabular}{l|c|c|}
\hline Estudio & $\begin{array}{c}\text { Sensibilidad } \\
(\mathrm{IC95} \%)\end{array}$ & $\begin{array}{c}\text { Especificidad } \\
(\mathrm{IC95} \%)\end{array}$ \\
\hline $\begin{array}{l}\text { Screening Tool for As- } \\
\text { sessment of Malnutrition } \\
\text { in Paediatrics (STAMP) }\end{array}$ & $\begin{array}{c}1.00 \\
(0.75,1.00)\end{array}$ & $\begin{array}{c}0.40 \\
(0.23,0.59)\end{array}$ \\
$\begin{array}{l}\text { Screening Tool for Risk } \\
\text { On Nutritional Status } \\
\text { and Growth (STRONG- } \\
\text { kids) }\end{array}$ & 0.95 & 0.52 \\
$\begin{array}{l}\text { Screening Tool for Risk } \\
\text { On Nutritional Status } \\
\text { and Growth (STRONG- } \\
\text { kids) }\end{array}$ & $(0.74,0.99)$ & $(0.46,0.57)$ \\
& & 0.39 \\
\end{tabular}

Tomado de: Huysentruyt, K, Devreker T, Dejonchheer J, De Schepper J, Vandenplas I, Cool F. The Accuracy of Nutritional Screening Tools in Assessing the Risk of Undernutrition in Hospitalized Children: A Systematic Review of Literature and Meta-analysis. J Pediatr Gastroenterol Nutr 2015;61(2):159-66.

D2. Bajo riesgo MÁS moderado vs alto riesgo

\begin{tabular}{|c|c|c|}
\hline Estudio & $\begin{array}{l}\text { Sensibilidad } \\
\text { (IC95\%) }\end{array}$ & $\begin{array}{c}\text { Especificidad } \\
\text { (IC95\%) }\end{array}$ \\
\hline $\begin{array}{l}\text { Screening Tool for As- } \\
\text { sessment of Malnutrition } \\
\text { in Paediatrics (STAMP) }\end{array}$ & $\begin{array}{c}0.85(0.55 \\
.98)\end{array}$ & $\begin{array}{c}0.73(0.54 \\
0.88)\end{array}$ \\
\hline $\begin{array}{l}\text { Screening Tool for Risk } \\
\text { On Nutritional Status } \\
\text { and Growth (STRONG- } \\
\text { kids) }\end{array}$ & $\begin{array}{c}0.43(0.27 \\
0.61)\end{array}$ & $\begin{array}{c}0.96(0.94 \\
0.98)\end{array}$ \\
\hline $\begin{array}{l}\text { Screening Tool for Risk } \\
\text { On Nutritional Status } \\
\text { and Growth (STRONG- } \\
\text { kids) }\end{array}$ & $\begin{array}{c}0.83(0.52 \\
0.98)\end{array}$ & $\begin{array}{c}0.94(0.81 \\
0.99)\end{array}$ \\
\hline
\end{tabular}

Tomado de: Huysentruyt, K, Devreker T, Dejonchheer J, De Schepper J, Vandenplas I, Cool F. The Accuracy of Nutritional Screening Tools in Assessing the Risk of Undernutrition in Hospitalized Children: A Systematic Review of Literature and Meta-analysis. J Pediatr Gastroenterol Nutr 2015;61(2):159-66. 
2. Guía de práctica clínica CENETEC. Desnutrición intrahospitalaria: tamizaje, diagnóstico y tratamiento. Evidencias y recomendaciones. Catálogo Maestro de Guías de Práctica Clínica IMSS;641-63.

3. Durakbaça ÇU, Fettahoğlu S, Bayar A, Mutus M, Okur $H$. The Prevalence of malnutrition and effectiveness of STRONGkids tool in the identification of malnutrition risks among pediatric surgical patients. Balkan Med J. 2014;31(4):313-21.

4. Mărginean $\mathrm{O}$, Pitea $\mathrm{AM}$, Voidăzan $\mathrm{S}$, Mărginean $\mathrm{C}$. Prevalence and assessment of malnutrition risk among hospitalized children in Romania. J Health Popul Nutr. 2014;32(1):97-102.

5. Velasco C, García E, Rodríguez V, Frias L, Garriga R, Álvarez $\mathrm{J}$, et al. Comparison of four nutritional screening tools to detect nutritional risk in hospitalized patients: a multicentre study. Eur J Clin Nutr. 2011 Feb;65(2):269-74.

6. Moeeni V, Day AS. Nutritional risk screening tools in hospitalized children. Int J Child Health Nutr. 2012;(1):39-43.

7. Hartman C, Shamir R, Hecht C, Koletzko B. Malnutrition screening tools for hospitalized children. Curr Opin Clin Nutr Metab Care. 2012;15(3):303-9.

8. Reilly HM, Martineau JK, Moran A, Kennedy H. Nutritional screening-evaluation and implementation of a simple nutrition risk score. Clin Nutr. 1995;14(5):269-73.
9. Sermet-Gaudelus I, Poisson-Salomon AS, Colomb V, Brusset MC, Mosser F, Berrier F, et al. Simple pediatric nutritional risk score to identify children at risk of malnutrition. Am J Clin Nutr. 2000;72(1):64-70.

10. Secker DJ, Jeejeebhoy KN. Subjective global nutritional assessment for children. Am J Clin Nutr. 2007;85(4):1083-9.

11. McCarthy H, McNulty H, Dixon M, Eaton-Evans MJ. Screening for nutrition risk in children: the validation of a new tool. Hum Nutr Diet J. 2008:21(4):395-96.

12. Hulst JM, Zwart H, Hop EC, Joosten KFM. Dutch national survey to test the STRONG kids nutritional risk screening tool in hospitalized children. Clin Nutr. 2010;29(1):106-11.

13. Gerasimidis K, Keane O, Macleod I, Flynn DM, Wright CM. A four-stage evaluation of the Paediatric Yorkhill Malnutrition Score in a tertiary paediatric hospital and a district general hospital. Br J Nutr. 2010;104(5):751-6.

14. Huysentruyt K, Devreker T, Dejonchheer J, De Schepper J, Vandenplas I, Cool F. The accuracy of nutritional screening tools in assessing the risk of under-nutrition in hospitalized children: A systematic review of literature and metaanalysis. J Pediatr Gastroenterol Nutr. 2015;61(2):159-66.

15. Pinilla G. Evaluación de la atención nutricional en el paciente pediátrico hospitalizado en el INP. Tesis de la especialidad. UNAM, Ciudad de México, 2016.

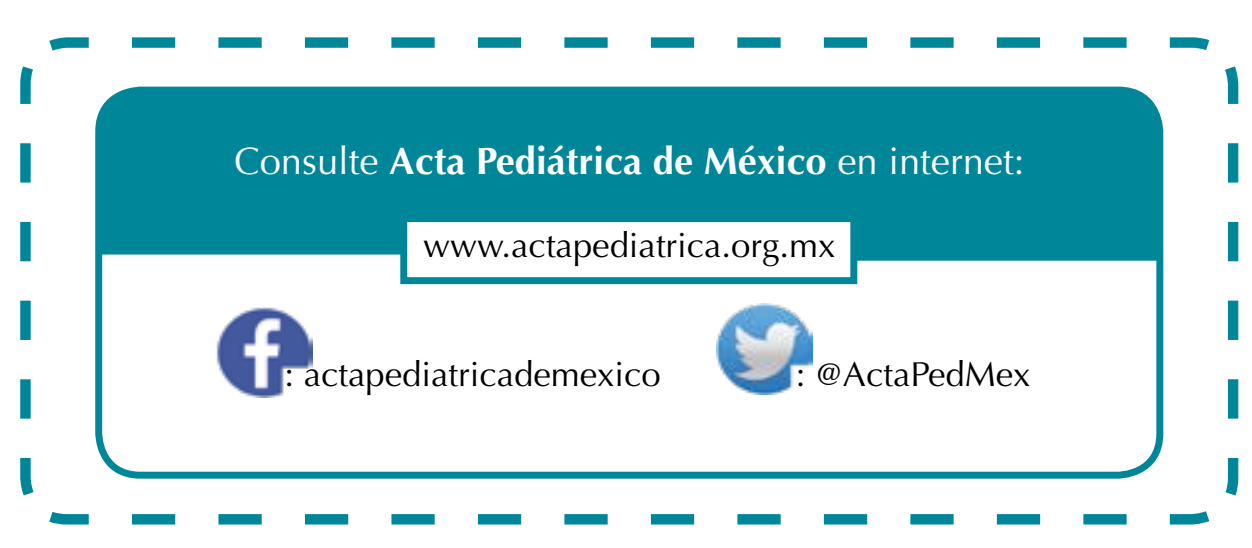

